\title{
Difficulties Encountered by Arabic-Speaking Undergraduate and Graduate English Language Students in Interpreting English Formulaic Expressions
}

\author{
Ayman Nazzal ${ }^{1}$, Fayez Aqel ${ }^{1} \&$ Abdul Kareem Igbaria $^{1}$ \\ ${ }^{1}$ Faculty of Human Sciences, Department of English, An-Najah National University, Nablus, Palestine \\ Correspondence: Abdul Kareem Igbaria, The Department of English, An-Najah National University, Nablus, \\ Palestine. E-mail: kareem1@netvision.net.il
}

\author{
Received: May 14, 2014 Accepted: June 23, 2014 Online Published: October 1, 2014 \\ doi:10.5539/ijel.v4n5p42 URL: http://dx.doi.org/10.5539/ijel.v4n5p42
}

\begin{abstract}
This study investigates the difficulties that undergraduate and graduate students of English language encounter in their interpretation and translation of English idiomatic/formulaic expressions into Arabic. Since the majority of these idiomatic expressions (referred to hereafter as IEs) in English or any other language potentially have more than one interpretation, it has been assumed that these expressions constitute a major problem for non-native speakers of English, particularly for those who do not have adequate semantic and pragmatic competence in the target culture.

The interpretation/translation task used in this study consists of three English formulaic expressions deliberately selected to measure both undergraduate and graduate students' semantic and pragmatic competence in interpreting/translating these formulaic expressions. The results of this study are based on the written interpretation/translation and the informal solicitation of responses from 83 undergraduate students of English language and 13 graduate students of Applied Linguistics and Translation.

The disparity in the students' performance on the interpretation task that was administered to both groups unequivocally verified the claim that 'inter-lingual transfer' occurs when foreign students are called upon to translate from their mother tongue to a foreign language; and that acquiring adequate competence in the pragmatics of the target language and culture is highly essential for the acquisition of literacy and avoidance of misinterpretation of such expressions (Gass \& Selinker, 1983; Odlin, 1989; Kharma \& Hajjaj, 1997; Mahmoud, 2002).

The findings of this study indicate that graduate students have done overwhelmingly well in comparison with their undergraduate counterparts. This is probably due to their continued training in translating material to and from the target language and culture. The findings have also emphasized the importance of providing students with adequate training in pragmatics, intercultural communication, and translation.
\end{abstract}

Keywords: intercultural pragmatic, interpretation, formulaic/idiomatic expressions

\section{Introduction}

In his famous book entitled "Formulaic Language and the Lexicon", Wray (2002) remarked that when some native speakers were asked about their perception and interpretation of what the formulaic expression 'rice crispy' really means, they were shocked to realize that it meant no more and no less than its conceptual content: This cereal is made from rice and it is crispy and delicious. It has been reported that the majority of respondents were under the impression that this formulaic expression (referred to hereafter as IEs) had meant something other than what the conceptual content of this phrase itself really means.

The most revealing and insightful aspect of the respondents' perception of this particular formulaic expression is the level of difficulty that some of these formulaic/idiomatic expressions can pose to native speakers of English. In addition, this particular situation can be considered a litmus test in the sense that it reveals the type of disparity which manifests itself in the perception of native speakers of one formulaic expression when the expectation is that there has to be some consistency in their perception of this particular formulaic expression. If the perception of some native speakers of this formulaic expression reveals some variation in their responses, the 
perception and interpretation of IEs are liable to be an even more challenging task for non-native speakers of English. The primary focus of the present study is to emphasize the type of difficulties that non-native speakers of English encounter in their translation/interpretation of IEs and their consequences.

There is no doubt that there are various reasons that account for the difficulties foreign students encounter in translating or interpreting IEs. These are mainly inherent in the type of IEs themselves or due to the lack of adequate pragmatic competence of such expressions. For example, one of the difficulties stems from the fact that the meaning of such IEs cannot be derived from the meaning of their individual words. For instance the IE "the bowel is lost", which can be used literary/conceptually, is idiomatically used to mean "anarchy reigns".

The disparity between the semantic and pragmatic meaning of this particular IE is too broad and difficult for foreign students with inadequate pragmatic competence and practice to account for. Other IEs may constitute a real challenge to foreign students because their conceptual meaning is not used literally but rather idiomatically. An example of this is the IE "Once in a blue moon". Other IEs have to be used in the plural rather than the singular form to avoid losing their idiomatic connotation or meaning. For example, we normally say "Kill the goose that laid the golden egg", but not "Kill the geese that laid the golden eggs". However, other IEs occur in the plural form such as: "Change horses in the middle of a stream" rather than " Change a horse in the middle of a stream" , or put one's eggs in one basket", but not "put one's egg in one basket (Urdang \& Abate, 1983).

Other IEs pose great difficulty to students because some types of IEs require a fixed order of individual words. For instance, we say "My flesh and blood" rather than "My blood and flesh." In addition, the real or intended meaning of this idiom is different than the literal meaning of its individual words.

The difficulty is further compounded by the fact that the literal interpretation of these IEs will not always contribute to the intended meaning of most IEs. There is a tendency on the part of native speakers to use them often in formal and informal conversation. Consequently, mastering them is a key to demonstrating proficient command of the language and an ability to converse with native speakers as intelligibly as possible.

It has been said that the difficulties of these IEs lie in the fact that they belong to different categories, and each category requires a specific translation approach to account for its adequate equivalence in the target language. This obviously compounds and complicates the problem for non-native speakers of English. Unless they develop adequate pragmatic competence of the target language, they are destined to misconstrue native speakers and risk running into a communication breakdown.

\subsection{The Purpose of the Study}

The study was conducted on both undergraduate and graduate students of English language and translation to test existing hypotheses regarding the role of pragmatic differences and how students of English language deal with expressions that require distinct competences in both linguistics and pragmatics. We can consider this study to be a genuine experiment since its scope and number of subjects was 100 college students. The study will provide important insights and feedback on the pivotal question which the researchers attempt to underlie its consequences on participants in cross/intercultural communication encounters in this preliminary research paper.

\subsection{Significance of the Study}

The importance of the study of IEs lies in the fact that while native speakers have a tendency to use them often during social interaction, there is inadequate effort allocated to the enhancement of students' pragmatic competence in undergraduate education. In fact, it has been said that IEs are the means with which people express their traditions of social reality, cultural values, and the implementation of culture-specific actions. This makes IEs language-specific despite the possibility of finding their equivalents in other languages. In addition IEs are language particular since a specific idiom in a particular language may or may not have an equivalent in others. For instance the idiom 'twist his arm' has equivalence in Arabic. Consequently the expectation is that English major students would not have a problem dealing with this type of idioms. On the other hand, an idiom such as 'a straw man' would constitute a problem, since it has no equivalence in Arabic. English is replete with IEs that are identical, semi-identical, different, or semi-different than those in Arabic. This makes the study of IEs extremely important for speakers of other languages in order to acquire adequate pragmatic competence in English.

The importance of this study also lies in emphasizing the difficulty speakers of English as a foreign language experience in encountering IEs because comprehending some IEs requires both semantic and pragmatic competence. Formal and informal intercultural or intra-cultural conversations and communication are a rich matrix for such IEs. Native speakers tend to use IEs frequently due to their aesthetic value, charm, color, and flavor that they contribute to language. 
Finally, this study is important since it is the first that aims at emphasizing the types of difficulties that non-native speakers of English face in their translation of English IEs and their consequences. It also attempts to pinpoint the discrepancies between undergraduate and graduate student at An-Najah National University.

\subsection{Research Hypotheses}

The researchers hypothesize that

1) There are no statistically significant differences $(\alpha=0.05)$ between the ratio of undergraduate students and graduate students in the recognition of the $(\mathrm{C})$ meaning of expression 1.

2) There are no statistically significant differences $(\alpha=0.05)$ between the ratio of undergraduate students and graduate students in the recognition of the (D) meaning of expression 1.

3) There are no statistically significant differences $(\alpha=0.05)$ between the ratio of undergraduate students and graduate students in the recognition of the $(\mathrm{C})$ meaning of expression 2.

4) There are no statistically significant differences $(\alpha=0.05)$ between the ratio of undergraduate students and graduate students in the recognition of the (D) meaning of expression 2.

5) There are no statistically significant differences $(\alpha=0.05)$ between the ratio of undergraduate students and graduate students in the recognition of the (C) meaning of expression 3.

6) There are no statistically significant differences $(\alpha=0.05)$ between the ratio of undergraduate students and graduate students in the recognition of the (D) meaning of expression 3.

\section{Literature Review and Theoretical Grounds}

Theories of meaning suggest that concepts are units that are structured and complex. Concepts comprise three entries: the lexical entry, the logical entry, and the encyclopedic entry. However, formulaic forms do not conform to this standard pattern (Sperber \& Wilson, 1995). Formulaic expressions include greetings, proverbs, warnings, notices, idioms, etc. and such terms are usually rendered holistically in translation.

Research has been conducted in the area of formulaic expressions and translation. In his study of Galician formulaic forms, Sequeiros (2004) explored the characteristics of formulaic expressions and discussed how these forms are translated. He also showed that the translation of such expressions reveals the difficulties of their translation and demonstrated how diverse the linguistic forms are in human communication. He maintains that: [Formulaic] "Forms are, from a linguistic point of view, radically different from language to language and in most cases literal linguistic translation does not work". Normally there is only a limited set of forms for each type of expression that may or may not be available (ibid: 105)'. He concluded by claiming that formulaic expressions cannot be translated via using the equivalence approach and in order to translate these expressions we should consider both their conceptual nature and the way their interpretation derives from general pragmatic processes.

Wray (2002) maintains that mastery of idiomatic expressions is a significant component of successful language learning. Other researchers attempted to foreground formulaic forms in teaching syllabuses (Willis, 1990; Natting \& DeCarrico, 1992; Lewis 1993). The researchers, regardless of the different points of view they hold about the relation of these words to the learners' accumulation of lexical and grammatical knowledge, concluded that such forms "can, and should, be perceived by the learners and the teachers in terms of their component parts".

Pawley and Syder (1983) claim that formulaic sequences, even though they consist of a sequence of individual words, can be processed more easily and quickly than single memorized units. By comparing the reading times for formulaic forms and non-formulaic ones for both native and nonnative speakers, Conklin and Schmitt (2006) found that the former forms were read more quickly by both groups of participants.

In his study "The Equivalence and Translatability of English and Arabic Idioms" Awwad (1991) maintains that idiomatic forms-idioms are problematic areas for translators and foreign language teachers. He investigated the types, context of occurrence, and discussed the constraints idioms impose on translating from English and Arabic. Furthermore, he pointed out the problems of misinterpretation and cultural differences encountered in translation. He proposed a theoretical framework for the translation of idioms that assigns an idiom to one of four categories of correspondence between English and Arabic in a hierarchy of difficulty.

The findings of previous studies on Arab students' acquisition of English language point out the tendency of Arab students to transfer idiomatic expression from Arabic to English because they do not possess an adequate level of language proficiency to allow them to use and understand English idiomatic expressions (Baker, 1992; 
Mahmoud, 2002; Kharma \& Hajjaj, 1997). The findings of these studies emphasize the claims that Arab students' lack of knowledge and practice in the foreign language they are acquiring undermines their ability to use idioms in intercultural communication encounters.

It is obvious that the difficulties in accounting for the interpretation or translation of these IEs stem from non-native speakers' failure to comprehend either or both the conceptual (semantic) and non-conventional meaning (pragmatic) of a particular IE. Another primary cause for such a failure occurs when there is no equivalent of such an expression in the students' native language. In such instances, it becomes extremely difficult for students to account for such expressions.

This emphasizes the assumptions of Kharma and Hajjaj (1997) concerning the transfer of idiomatic expressions from Arabic to English by Arab students learning English. One can note the occurrence of such a strong possibility by contrasting students' responses to 'piece of cake' with their responses to both 'hot stuff' and 'welcome aboard. One can easily note that students' responses to the expression 'piece of cake' were overwhelmingly satisfactory in comparison with the last two expressions and that is due to the fact that the idiom 'piece of cake' has its equivalent expression in Arabic whereas 'welcome aboard', and 'hot stuff' have no equivalents.

The difficulties that non-native speakers experience in their interpretation of formulaic expression also augments Hymes' $(1971,1974)$ notion of the importance of acquiring 'communicative competence' in the target culture. Such competence enables non-native speakers to learn the rules of language use and perception in a variety of social contexts.

Thomas (1983) also claims that cross-cultural failure occurs as a result of perceiving a particular message differently as a consequence of paying a great deal of attention to the semantic import, thus sacrificing the pragmatic one. This seems to apply to the situation encountered by students dealing with IEs. Thomas (ibid) points out that while the distinction between them, i.e., the semantic versus the pragmatic meaning in some instances is too difficult to sort out, cross-cultural pragmatic failures occurs either in instances where the speaker or interpreter fails to convey the 'illocutionary force' or intended meaning of his/her utterance (Austin, 1962, 1975; Searle, 1979) or in an instance in which the speaker's interpretation or translation of a message from or into the target language is subjected to relying or using native-like strategies.

Since the process of translation of such formulaic/idiomatic expressions is grounded in both language and culture, it would be erroneous to ignore the influence of language and culture on the translator engaged in either process. On this note the question of translation of such idiomatic expressions seems to invoke the discussion of several major perspectives on the same subject one of which pertains to the perspective or view that shows the profound influence of language on the perception of its speakers. This perspective has been articulated extensively by many prominent scholars and linguists, primarily by Edward Sapir (1921), who posed the "Relativity Hypothesis". This hypothesis emphasizes that the striking differences among languages are destined to have an impact on the perception of their speakers. This implies that the organization and structure of a particular language is very likely to make its speakers conceive things under the influence of the structure of that language.

Even though Sapir's hypothesis has received a great deal of criticism upon its initiation and formation, it has lots of merits in terms of being able to account for some of the communication break down which results in inter-cross-cultural communication situations. One of the advantages which this hypothesis offers deals with the concept of 'habitual thoughts' which people employ in social interaction within a specific culture. For instance, the concept of 'color' or 'time' in an oriental culture varies from the same concept in an occidental culture. The keen linguist or ethnographer can detect this variation being reflected not only in the structuring of a particular language but also in the social behavior of the speakers of a particular language.

Therefore, language is likely to induce its speakers to see the social world of which they are a part in a particular way. This influence has prompted Sapir to make the following remarks on how language inclines its speakers to hold a particular social reality which differs from any other social reality of the speakers of any other language.

"No two languages are sufficiently similar to be considered as representing the same social reality. The worlds in which different societies live are distinct Worlds not merely the same world with different labels attached. (Sapir 1921: 69)

Sapir's comments are insightful and revealing provided that one has the competence to find out their validity and viability. These findings show several insightful observations one of which is the great influence of one's language on one's perception of the social world in which he/she lives. It also shows the difficulty that the interpreter faces upon handling the task assigned to him/her. Furthermore, these comments incline one to see the 
fragility of the situation in which interpreters and communicators find themselves. This leads one to deduce that it would be counter-productive to underestimate the impact and influence of a particular language on one's thinking and perception of the social world.

\section{Method}

\subsection{Subjects}

This study was conducted among two groups of university students. The first group consisted of 83 undergraduate college students majoring in English language and literature. The majority were juniors and seniors. They are native Arabic speakers with adequate proficiency in English. The students have studied several courses in translation. They are homogenous in terms of age and linguistic background. The second group consisted of 13 graduate students in the M.A. program in Applied Linguistics and Translation at An-Najah University. The majority have completed most of their M.A. courses in the program. They are heterogeneous in terms of age, linguistic background and in their competence in English.

Table 1. Meanings of formulaic expressions and their symbols

\begin{tabular}{ll}
\hline Number of Formulaic expression & Meanings of Formulaic Expression \\
\hline 1. Piece of cake & C. Connotative \\
& D. Denotative \\
2. Welcome aboard & C. Connotative \\
& D. Denotative \\
3. hot stuff & C. connotative \\
& D. Denotative \\
\hline
\end{tabular}

\subsection{Data Collection}

This research paper emphasizes the types and consequences of the difficulties which students of English language encounter in translating English formulaic/idiomatic expressions and phrases. Three expressions were used to test students' understanding of IEs. The experiment/translation task consisted of three IEs that comprised component major part of a final exam administered to both undergraduate and graduate students of English language and Translation. This translation task was administered to 83 undergraduate and 13 graduate students at An-Najah National University in Nablus, Palestine.

This experiment constituted an important component of a final exam on a translation course for the entire academic semester of 2013. Therefore, students were told that they were being solicited for a particular study and answered all questions as part of their final academic exam. Students were asked to provide their own interpretation in Arabic or English of three formulaic expressions. Students were also told that these formulaic expressions might contain more than one interpretation in order to prevent confusion.

Students were also told that these expressions had two distinct meanings: a denotative or linguistic meaning and a connotative/pragmatic one. The formulaic expressions that were included in the exam were: 'a piece of cake', 'welcome aboard', and 'hot stuff'.

\section{Findings}

The results of students' interpretation and perception of these idiomatic/formulaic expressions have been analyzed and broken down into six tables for illustrating and interpretation the performance of both under and graduate students in English and translation.

The first Hypothesis: There are no statistically significant differences $(\alpha=0.05)$ between the ratio of undergraduate students and graduates students in the recognition of the (C) meaning of expression 1 (piece of cake).

To test the hypothesis a proportion test was used, and it yielded the following results as seen in table 2. 
Table 2. Piece of cake - C meaning (pragmatic import)

\begin{tabular}{lllll}
\hline Level & $\begin{array}{l}\text { No. of } \\
\text { correct answers }\end{array}$ & No. of students & Z value & Significance level \\
\hline U.G & 79 & 83 & 2.05 & $* 0.040$ \\
G & 13 & 13 & & \\
\hline
\end{tabular}

Significant at $(\alpha=0.05)$

The above table shows that there are statistically significant differences between the two groups of respondents in the recognition of meaning $\mathrm{C}$ of expression 1 in favor of the graduate students. This means that the first hypothesis was refuted.

The second hypothesis: There are no statistically significant differences at $(\alpha=0.05)$ between the ratio of undergraduate students and graduate students in the recognition of the (D) meaning of expression 1.

To test the hypothesis, a proportion test was used, which yielded the following results as seen in table 3 .

Table 3. Piece of cake - D meaning (semantic import)

\begin{tabular}{lllll}
\hline Level & $\begin{array}{l}\text { No. of } \\
\text { correct answers }\end{array}$ & No. Of students & Z value & Significance level \\
\hline U.G & 51 & 83 & 7.22 & $* 0.000001$ \\
G & 13 & 13 & & \\
\hline
\end{tabular}

Significant at $(\alpha=0.05)$

he above table shows that there are statistically significant differences between the two groups of respondents in the recognition of meaning $\mathrm{C}$ of expression 1 in favor of the graduate students. This means that that the first hypothesis was refuted.

The third Hypothesis states that there are no statistically significant differences at $(\alpha=0.05)$ between the ratio of undergraduate students and graduate students in the recognition of the (C) meaning of expression 2.

To test the hypothesis, a proportion test was used, which yielded the following results as seen in the following table.

Table 4. Welcome aboard - C meaning

\begin{tabular}{lllll}
\hline Level & $\begin{array}{l}\text { No. of } \\
\text { correct answers }\end{array}$ & No. of students & Z value & Significance level \\
\hline U.G & 33 & 83 & 1.50 & 0.134 \\
G & 8 & 13 & & \\
\hline
\end{tabular}

Significant at $(\alpha=0.05)$

The above table shows that there are statistically significant differences between the two groups of respondents in the recognition of meaning $\mathrm{C}$ of expression 1 in favor of the graduate students. This means that that the first hypothesis was refuted.

The fourth Hypothesis which states that there are no statistically significant differences at $(\alpha=0.05)$ between the ratio of undergraduate students and graduate students in the recognition of the (D) meaning of expression 2.

To test the hypothesis, a proportion test was used, which yielded the following results as seen in table 5 .

Table 5. Welcome aboard - D meaning

\begin{tabular}{lllll}
\hline Level & $\begin{array}{l}\text { No. of } \\
\text { correct answers }\end{array}$ & No. of students & Z value & Significance level \\
\hline U.G & 25 & 83 & 4.86 & $* 0.000001$ \\
G & 11 & 13 & & \\
\hline
\end{tabular}

Significant at $(\alpha=0.05)$ 
The above table shows that there are statistically significant differences between the two groups of respondents in the recognition of meaning $\mathrm{C}$ of expression 1 in favor of the graduate students. This means that that the first hypothesis was refuted.

The fifth Hypothesis states that there are no statistically significant differences at ( $\alpha=0.05$ ) between the ratio of undergraduate students and graduate students in recognition the (C) meaning of expression 3.

To test the hypothesis a proportion test was used which yielded the following results as seen in table 6 .

Table 6. Hot stuff - C meaning

\begin{tabular}{lllll}
\hline Level & $\begin{array}{l}\text { No. of } \\
\text { correct answers }\end{array}$ & No. of students & Z value & Significance level \\
\hline U.G & 25 & 83 & 6.95 & $* 0.000001$ \\
G & 12 & 13 & & \\
\hline
\end{tabular}

Significant at $(\alpha=0.05)$

The above table shows that there are statistically significant differences between the two groups of respondents in the recognition of meaning $\mathrm{C}$ of expression 1 in favor of the graduate students. This means that the first hypothesis was refuted.

The Sixth Hypothesis states that there are no statistically significant differences at $(\alpha=0.05)$ between the ratio of undergraduate students and graduate students in the recognition of the (D) meaning of expression 3. To test the hypothesis, proportion test was used, and it yielded the following results as seen in table 7 .

Table 7. Hot stuff - D meaning

\begin{tabular}{lllll}
\hline Level & $\begin{array}{l}\text { No. of } \\
\text { correct answers }\end{array}$ & No. of students & Z value & Significance level \\
\hline U.G & 33 & 83 & 5.75 & $* 0.000001$ \\
G & 12 & 13 & & \\
\hline
\end{tabular}

Significant at $(\alpha=0.05)$

The above table shows that there are statistically significant differences between the two groups of respondents in the recognition of meaning $\mathrm{D}$ of expression 1 in favor of the graduate students. This means that the first hypothesis was refuted.

\section{Analysis and Discussion}

The findings of this study have been insightful and revealing in pointing out the type of difficulties which university students encounter in the interpretation/translation of English idiomatic expressions for the attainment of both the denotative (or semantic) and connotative (or pragmatic) meaning. In fact, the findings of this study emphasize the importance of pragmatic competence. The majority of students made more mistakes pertaining to connotative matters than errors pertaining to semantic or linguistic matters.

The first observation which we can deduce from the statistical results is that there is a wide and conspicuous disparity between the two groups in all their responses. For example most graduate students were able to attend to both meanings successfully with regard to the three formulaic expressions. However, there were two graduate students out of the 13 who failed to supply the pragmatic interpretation of the expression "welcome aboard', and only one graduate student was not able to provide the pragmatic interpretation of the expression 'hot stuff'. In contrast, undergraduate students' responses were less satisfactory than those of the graduate students. This can be attributed to the assumption that their exposure to the use of these formulaic expressions in real-life situations is infrequent.

The discussion of the findings of each expression is provided below.

\subsection{Piece of Cake}

In the case of 'piece of cake', the situation has taken a different direction. 51 out of 83 undergraduates have attended to the semantic import of the formulaic expression 'piece of cake' while 32 of them failed to supply the semantic import. There are obviously a considerable number of students who either have forgotten to provide the 
semantic import of this expression or have provided the pragmatic import instead. With regard to the graduate students, all of them have attended to the semantic import of this expression. This is not surprising given the fact that they are more fluent in the target language and its linguistic system than undergraduates.

Pragmatically, the disparity between undergraduates and graduates in providing the pragmatic import is extremely narrow to the point that both groups have successfully attended to this expression. For instance all 13 graduate students have been able to supply the pragmatic meaning of this expression. There were only 4 undergraduates who failed to attend to the pragmatic import of this expression. Therefore, one can note that the majority of students (79 undergraduate college students and 13 graduate students) have attended to the pragmatic import of this expression successfully and that is due to the fact that this particular idiom has its 'equivalence' in their native language.

This supports the assumptions of Kharma and Hajjaj (1997) concerning the transfer of idiomatic expressions from Arabic to English by Arab students learning English. One can note the occurrence of such a strong possibility by contrasting students' responses to 'piece of cake' with their responses to both 'hot stuff' and 'welcome aboard'. Students' responses to the expression 'piece of cake' were overwhelmingly satisfactory in comparison with the last two expressions and that is due to the fact that the idiom 'piece of cake' has its equivalence in Arabic whereas 'welcome aboard' and 'hot stuff' have no equivalent.

Ironically, students captured the pragmatic import of this expression, but a large number of them failed to provide its semantic import. Only 32 out of 83 were able to provide the semantic import of 'piece of cake'. This implies that students have forgotten to supply the semantic meaning even though they captured the pragmatic one easily. Alternately, students have had little exposure to this expression and some of them supplied the pragmatic meaning before the semantic one. The researchers are inclined to favor the first assumption that students attended to the pragmatic meaning and unwittingly forgotten the semantic one. The semantic import is highly facile and it is extremely unlikely that students could not account for it. In fact our solicitation of some students' responses ascertains our adopted belief that it was an oversight for some of them.

\subsection{Welcome Aboard}

The disparity between undergraduates and graduates regarding 'welcome aboard' is not broad, but is revealing in terms of the level of competence which graduate students possess in comparison with undergraduates. The findings point out that 33 undergraduates out of 83 were able to attended to the semantic import of this expression while 50 undergraduates were unable to provide an adequate interpretation of the linguistic content of this expression. However, graduate students' responses were overwhelmingly positive since the findings reveal that 11 out of 13 graduates were able to supply an adequate response to the semantic import of this expression.

Pragmatically, the disparity between these two groups is crystal clear and revealing in terms of the level of communicative competence which each group possesses over the other one. For example the statistical findings point out that 25 out of 83 undergraduates were able to supply a second interpretation for this expression, while 8 out of 13 graduate students who were able to provide a second interpretation to this expression.

In comparison with students' responses to the connotative meaning, only 21 students were able to provide an accurate interpretation of the expression 'welcome aboard'. This disparity in students' responses to this expression confirms the plausibility and soundness of the proposition that the crucial problem which confront our English major students has to do with their being unable to capture the pragmatic/connotative meaning since students have had little exposure to the target culture and therefore they are at disadvantage when they have to provide the connotative interpretation of any expression which happens to have more than one interpretation.

Students indicated that they had never heard of the second meaning of the expression 'welcome aboard' that deals with the instance of being welcomed to a particular social, political, religious group or affiliation. We are inclined to conclude that exposure to the use of formulaic expressions is of great importance to helping students account for the interpretation of these formulaic expressions, particularly those with double-meaning.

\subsection{Hot Stuff}

Students' responses regarding the expression 'Hot stuff' revealed a broad disparity. Only one graduate student out of 13 failed to provide the semantic import for the expression 'hot stuff'. On the other hand, findings reveal that 50 out of 83 undergraduates failed to attend to the semantic import and only 33 undergraduates were able to attend to this expression semantically. The disparity between the two groups is broad and revealing and shows the level of competence of one group over the other. 50 out of 83 undergraduate students failed to supply the semantic import of this formulaic expression in comparison with 1 out of 13 graduates. 
Only 25 out of 83 undergraduate students were able to provide the correct pragmatic/connotative interpretation of this expression. The remaining 58 failed to attend to the pragmatic import of the expression. In contrast, the majority of graduate students provided the pragmatic import of this expression. The statistical findings indicate that 1 out of 13 was not able to provide the proper pragmatic interpretation of the same expression. As a result, we can draw the following inferences:

Undergraduate English majors were not exposed previously to the exposure to the expression 'hot stuff', to the degree that their responses to both meanings were inadequate. The second inference which can be drawn from their responses is that this expression is the most difficult primarily for undergraduate students in contrast with the previous two formulaic expressions. Overall while most undergraduates have attended to the linguistic meaning of these three expressions, they were unable to satisfactorily provide the pragmatic interpretation of the same expressions particularly when dealing with 'welcome aboard', and 'hot stuff'.

This study has emphasized the soundness and viability of our proposition that the lack of pragmatic competence that our English language students manifest in their responses and interpretation is a crucial element in contributing to their failure to deal successfully with formulaic expression in translation or intercultural communication encounters. This study also emphasized the assumption that continued exposure of English language students to the target culture is highly essential for acquiring proper understanding of formulaic expressions. The initial discrepancy in the findings in this study between graduates' and undergraduates' responses to the same formulaic expressions reveals that undergraduates' pragmatic competence is less adequate than that of their graduate counterparts. This emphasizes the validity of the proposition that acquiring pragmatic competence in the target culture is a key element to an adequate understanding of intercultural communication or translating written or spoken material.

In dealing with the third expression, 'hot stuff', the statistical results were strikingly shocking since more than 50 undergraduate college students out of 83 were unable to provide either interpretation. Based on this particular observation, we can conclude that the disparity between graduate and undergraduate in attending to these formulaic expressions broad. Consequently, the type of pragmatic competence that graduate students possess far exceeds that of undergraduate college students. In addition, graduate students have more exposure to the use of this expression since it is part of their specialization.

\section{Conclusion}

The findings of this study emphasize the types of difficulties that students of linguistics and translation encounter in translating/interpreting formulaic or idiomatic expressions from English into Arabic. One of the major difficulties has to do with inability to distinguish between semantic and pragmatic imports - both of which exist in some idiomatic/formulaic expressions. As a result, the majority of students opted for the semantic import at the expense of the pragmatic one and in so doing their rendition or interpretation of a specific expression is pragmatically lacking.

The findings also pointed out that 'idiomatic expressions' that have no Arabic equivalent constitute a problem for college students. Statistical data has revealed that a majority of the students had less difficulty dealing with the expression 'piece of cake' which has an equivalent in Arabic. Finally, the findings emphasized the soundness and viability of the proposition that the lack of pragmatic competence that our English language students manifest in their responses and interpretation is a crucial element in contributing to their failure to deal successfully with formulaic expression in translation or intercultural communication encounters. Therefore, continued exposure to the target culture is highly essential for having proper understanding of formulaic expression. This can be accomplished by exposing students to the target culture through a technology-enhanced learning environment. The accessibility of the Internet to the majority of learners is an enhancing and contributing factor for students to acquire pragmatic literacy in the target culture.

\section{References}

Austin, J. L. (1962). How to do things with words. Cambridge: Harvard University Press.

Austin, J. L. (1975). How to do things with words. Cambridge: Harvard University Press. http://dx.doi.org/10.1093/acprof:oso/9780198245537.001.0001

Awwad, M. (1991). Equivalence and the translatability of English and Arabic idioms. PSiCL Vol .26: 57-67.

Baker, M. (1992). In other words: A Course on translation. London: Rutledge. http://dx.doi.org/10.4324/9780203327579 
Conklin, K., \& Schmitt, N. (2006). Formulaic Sequences: Are They Processed More Quickly than Nonformulaic Language by Native and Nonnative Speakers? Oxford: Oxford University Press.

Gass, S., \& Selinker, L. (1983). Language transfer. In F. Eppert (Ed.), Transfer and translation in Language Learning. Singapore: SEAMEO.

Hymes, D. (1971). Pidginization and creolization of languages. London: Cambridge University Press.

Hymes, D. (1974). Foundation in sociolinguistics: An Ethnographic approach. Philadelphia: University of Pennsylvania Press.

Kharma, N., \& Hajjaj, A. (1997). Errors among Arab speakers: Analysis and remedy. Beirut: Libraire du Liban.

Lewis, M. (1993). The lexical approach. Hove; England: Language Teaching Publications.

Mahmoud, A. (2002). The Inter-lingual errors of Arab students: A course book education English majors. Sultan Qaboos University, Unpublished manuscript

Odlin, T. (1989). Language transfer. Cambridge, NY: Cambridge University Press. http://dx.doi.org/10.1017/CBO9781139524537

Pawley, A., \& Hodgetts, F. S. (1983). Two puzzles for linguistic theory: Native like selection and native like fluency. In Richards \& Schmidt (Eds.), Language and communication (pp. 191-226). London: Longman.

Sapir, E. (1921). Language. New York: Harcourt Brace. Reprint Harvest Books.

Searle, J. (1979). Expression and meaning. Cambridge: Cambridge University Press. http://dx.doi.org/10.1017/CBO9780511609213

Sequeiros, R. X. (2004). Formulaic expressions in translation. TRANS: Revista de traductología, 8, 105-113.

Sperber, D., \& Wilson, D. (1995). Post face to the second edition of Relevance: Communication and Cognition. Oxford: Blackwell.

Thomas, J. (1983). Cross-cultural pragmatic failure. Applied Linguistics, 4(2), 91-112. http://dx.doi.org/10.1093/applin/4.2.91

Willis, D. (1990). The lexical syllabus: A new approach to language teaching. London: Collins ELT.

Wray, A. (2002). Formulaic language and the lexicon. Cambridge: Cambridge University Press. http://dx.doi.org/10.1017/CBO9780511519772

\section{Copyrights}

Copyright for this article is retained by the author(s), with first publication rights granted to the journal.

This is an open-access article distributed under the terms and conditions of the Creative Commons Attribution license (http://creativecommons.org/licenses/by/3.0/). 